\title{
Comparative Evaluation of Conventional Blood Culture with Bactec 9050 for Bacterial Isolates in Clinically Suspected Cases of Fever of Unknown Origin
}

\author{
Avneet Kaur ${ }^{1}$, Puneet Singh Soodan ${ }^{2}$, Varsha A Singh ${ }^{3}$ \\ ${ }^{I}$ (Microbiology, M.M.Institute of Medical Sciences and Research , Mullana, Ambala/ Maharishi \\ Markandeshwar University, India) \\ ${ }_{2}^{2}$ (Dermatology, Shri Ram Murti Smarak institute of Medical Sciences, Barielly (U.P)/Rohilkhand \\ University, India) \\ ${ }_{3}^{3}$ (Microbiology, M.M.Institute of Medical Sciences and Research , Mullana, Ambala/ Maharishi \\ Markandeshwar University, India)
}

\begin{abstract}
:
Background: Patients with fever of unknown origin (FUO) are elusive and challenging clinical cases. Timely detection and identification of blood borne pathogen is one of the most important functions of microbiology laboratory.

Objectives: To evaluate the efficacy of BACTEC 9050 in comparison to conventional blood culture for detection of bacterial isolates in clinically suspected cases of fever of unknown origin.

Material and methods: Blood samples from 100 suspected cases of fever of unknown origin (FUO) were subjected to conventional blood culture and BACTEC 9050 culture system.

Results: Out of 100 suspected cases, $46 \%$ cases were culture positive with $80.43 \%$ pathogenic bacterial isolates comprising of $54.05 \%$ gram positive and $45.94 \%$ gram negative isolates. Predominant gram positive isolates were 35\% coagulase negative Staphylococcus and 30\% Staphylococcus aureus. Gram negative isolates were $29.41 \%$ Salmonella typhi followed by $17.64 \%$ E coli. BACTEC 9050 was observed to be sensitive (100\% ) as compared to conventional blood culture (67.56\%). Mean time to detection of significant pathogens was significantly less with the BACTEC 9050 than with conventional media.
\end{abstract}

Conclusion: BACTEC 9050 proved as a reliable and fast method to identify the blood stream pathogens in blood culture as compared to conventional culture methods.

Keywords: BACTEC 9050-Becton Dickinson Microbiology Systems, FUO-fever of unknown origin

\section{Introduction}

Pyrexia of unknown origin (PUO) continues to be one of the most challenging situations facing the physician. ${ }^{1}$ It is identified as a syndrome of fever that does not resolve spontaneously and in which the cause remains elusive even after an extensive diagnostic workup. Petersdorf and Beeson first coined the term fever of unknown origin in 1961 and explicitly defined it as (1) Temperature $>38.3^{\circ} \mathrm{C}\left(101^{\circ} \mathrm{F}\right)$ on several occasions (2) duration of fever of more than 3 weeks and (3) failure to reach to diagnosis despite one week of inpatient investigations. ${ }^{2}$ The differential diagnosis for PUO comprises over 200 disorders and is among the longest of any condition in medicine ${ }^{3}$ In adults, infections and cancer account for about $25-40 \%$ cases each of PUO. ${ }^{4}$ Autoimmune disorders account for rest of the 10-20\%cases. ${ }^{5}$ In children, $30-50 \%$ cases are due to infections, 5$10 \%$ cancer and remaining 10-20\% cases are due to autoimmune disorders. A cause is never identified in a significant proportion $(19 \%)$ of patients. ${ }^{6}$ Infectious disease is one of the leading causes of morbidity and mortality in most developing countries. A meticulous history, a thorough physical examination, discriminative use of investigative procedures and constant reassessment of all the parameters reveal the cause of the patients fever. ${ }^{7}$ Despite recent developed techniques, like nucleic acid probes, PCR and other molecular techniques for microbiological diagnosis, blood culture still remains the most practical and reliable method in the diagnosis of bloodstream infections. ${ }^{8}$ Blood cultures provide the best yield for microbiological diagnosis, with sensitivity ranging from 53 to $90 \% .^{9}$ Instrumentation of blood culture has accomplished rapidness, accuracy and cost effectiveness. It also eliminates cross contamination of cultures during repeated subculture. The BACTEC 9000 series of blood culture systems are fluorogenic, automated, non-invasive blood culture system designed for processing three to five blood cultures per day. ${ }^{10}$ Conventional method includes culture for two weeks to detect slow growing organisms and on special media if necessary. Hence our study was an attempt to evaluate the capability, efficiency and reliability of BACTEC 9050 in comparison to conventional blood culture for detection of bacterial isolates in clinically suspected cases of fever of unknown origin. 


\section{Material \& Methods:}

This cross-sectional study was carried out during the period January 2011 to December 2011 at M.M.Institute of Medical Sciences and Research, Mullana. Blood samples from 100 suspected cases of fever of unknown origin (FUO) attending the OPD and indoor were included in the study. Diagnosed cases of fever were excluded.

\subsection{Sample Collection And Processing:}

$10 \mathrm{ml}$ blood was collected aseptically from adult patients and was divided equally into BACTEC blood culture vial (aerobic) and conventional blood culture bottle containing $50 \mathrm{ml}$ of brain heart infusion broth (Dilution 1:10). ${ }^{11}$ For paediatric patients, $2 \mathrm{ml}$ of blood was collected and equally transferred into the BACTECTM PEDS PLUS/F vial and Conventional blood culture bottle containing $10 \mathrm{ml}$ of brain heart infusion broth. ${ }^{12}$ The inoculated BACTEC vials and conventional blood culture bottles were transported to the laboratory immediately.

The BACTEC bottle was placed into the BACTEC 9050. On receiving positive signals, bottles were removed and an aliquot of the broth was gram stained and subcultured onto the Blood agar, Mac Conkey's agar and Chocolate agar. Organism were identified by battery of biochemical reaction and all bottles were incubated for a minimum of 7 days before labelling as negative as per the manufacturer's protocol. ${ }^{10}$ All negative bottles were subcultured to chocolate agar plates and incubated aerobically at the end of the incubation period.Conventional technique was done by inoculating blood into blood culture bottles containing brain heart infusion broth. The bottles were incubated at $37^{\circ} \mathrm{C}$ and were shaken periodically. On 3rd, 5th, and 7th day, subcultures were done on Blood agar, Mac Conkey's agar and Chocolate agar. Futher processing was done by standard laboratory procedures. ${ }^{13}$ The negative blood sample was discarded after 7 days of incubation.

\section{Result}

The present study was a cross sectional study carried out at Bacteriology division in the Department of Microbiology at M.M.Institute of Medical Sciences \& Research, Mullana over a period of one year from January 2011 to December 2011. In this study an attempt has been made to compare BACTEC 9050 with Conventional blood culture for detecting pathogenic bacterial isolates.

Table 1: Rate of culture positivity in suspected patients of Fever of unknown origin

\begin{tabular}{|c|c|c|}
\hline Total $(\mathbf{n})$ & Culture positive $\mathbf{n}(\boldsymbol{\%})$ & Sterile $\mathbf{n}(\boldsymbol{\%})$ \\
\hline 100 & $46(46)$ & $54(54)$ \\
\hline
\end{tabular}

Out of total of 100 samples of fever of unknown origin culture positivity was seen in $46(46 \%)$ cases and 54 were sterile cases $(54 \%)$ by either of the two methods i.e. conventional blood culture and BACTEC 9050 .

Table 2: Distribution of Blood culture isolates on the basis of pathogenicity

\begin{tabular}{|c|c|c|}
\hline Total (n) & Pathogenic isolates n (\%) & Non pathogenic isolates n (\%) \\
\hline 46 & $37(80.43)$ & $9(19.56)$ \\
\hline
\end{tabular}

Out of 46 culture positive isolates, $37(80.43 \%)$ isolates were pathogenic while remaining $9(19.56 \%)$ were non pathogenic isolates.

Table 3 : Distribution of pathogenic organisms in Blood culture

\begin{tabular}{|c|c|c|}
\hline Total (n) & $\begin{array}{c}\text { Gram positive } \\
\text { n (\%) }\end{array}$ & $\begin{array}{c}\text { Gram negative } \\
\text { n(\%) }\end{array}$ \\
\hline 37 & $20(54.05)$ & $17(45.94)$ \\
\hline
\end{tabular}

Among the pathogenic isolates, 20 isolates (54.05\%) were gram positive while remaining 17 isolates (45.94\%) were found to be gram negative.

Table 4: Distribution of Pathogenic Gram positive organism

\begin{tabular}{|c|c|c|c|c|c|}
\hline $\begin{array}{c}\text { Total } \\
(\mathbf{n})\end{array}$ & $\begin{array}{c}\text { coagulase negative } \\
\text { Staphylococcus } \\
\mathbf{n}(\boldsymbol{\%})\end{array}$ & $\begin{array}{c}\text { Staphylococcus aureus } \\
\mathbf{n}(\boldsymbol{\%})\end{array}$ & $\begin{array}{c}\text { Streptococcus } \\
\text { pneumonia } \\
\mathbf{n}(\boldsymbol{\%})\end{array}$ & $\begin{array}{c}\text { Enterococcus } \\
\mathbf{n}(\%)\end{array}$ & $\begin{array}{c}\text { Candida } \\
\mathbf{n}(\boldsymbol{\%})\end{array}$ \\
\hline 20 & $7(35)$ & $6(30)$ & $3(15)$ & $2(10)$ & $2(10)$ \\
\hline
\end{tabular}

Out of 20 gram positive cases, 7 (35\%) were coagulase negative Staphylococcus followed by 6 (30\%) Staphylococcus aureus, 3(15\%) were Streptococcus pneumonia, Enterococcus 2(10\%) and Candida 2(10\%). 
Table 5: Distribution of Pathogenic Gram negative organism

\begin{tabular}{|c|c|c|c|c|c|c|}
\hline $\begin{array}{c}\text { Total } \\
(\mathbf{n})\end{array}$ & $\begin{array}{c}\text { S.typhi } \\
\mathbf{n}(\boldsymbol{\%})\end{array}$ & Ecoli n(\%) & $\begin{array}{c}\text { Pseudomonas n } \\
(\boldsymbol{\%})\end{array}$ & $\begin{array}{c}\text { Klebsiella n } \\
(\boldsymbol{\%})\end{array}$ & $\begin{array}{c}\text { Acinetobacter } \\
\mathbf{n}(\boldsymbol{\%})\end{array}$ & $\begin{array}{c}\text { Citrobacter n (\%) } \\
\text { Brucella n } \\
(\boldsymbol{\%})\end{array}$ \\
\hline 17 & $5(29.41)$ & $3(17.64)$ & $3(17.64)$ & $3(17.64)$ & $1(5.88)$ & $1(5.88)$ \\
\hline
\end{tabular}

Out of 17 cases, $5(29.41 \%)$ were Salmonella typhi followed by E coli 3(17.64\%), Pseudomonas 3(17.64\%), Klebsiella 3(17.64\%), Acinetobacter 1(5.88\%), Citrobacter 1(5.88\%) and Brucella 1(5.88\%).

Table 6: Distribution of culture positive cases according to Gender, Age and Residence

\begin{tabular}{|c|c|c|}
\hline Parameters & $\begin{array}{c}\text { Culture positive } \\
(n=46)\end{array}$ & $(\%)$ \\
\hline \multicolumn{3}{|l|}{ Gender } \\
\hline Male & $30 *$ & 65.22 \\
\hline Female & 16 & 34.78 \\
\hline \multicolumn{3}{|l|}{ Age } \\
\hline$<20$ & $24 *$ & 52.17 \\
\hline $21-30$ & 6 & 13.04 \\
\hline $31-40$ & 10 & 21.73 \\
\hline $41-50$ & 4 & 8.69 \\
\hline$>50$ & 2 & 4.34 \\
\hline \multicolumn{3}{|l|}{ Social status } \\
\hline Urban & 16 & 34.78 \\
\hline Rural & $30 *$ & 65.22 \\
\hline
\end{tabular}

*indicates 1 positive sample of Brucella.

In present study, males constituted majority (65.22\%) of the patients from rural background (65.22\%). No definite correlation between occupation and culture positivity was observed .Maximum patients were found in the younger age group of less than 20 years $(52.17 \%)$ followed by $31-40$ years $(21.73 \%), 21-30$ years $(13.04 \%), 31-40$ years $(8.69 \%)$ and $>50$ years of age $(4.34 \%)$.

Table 7: Total time of detection of Bacterial isolates by BACTEC 9050

\begin{tabular}{|c|c|c|c|c|}
\hline Type of organism & No of samples $\mathbf{n}(\boldsymbol{\%})$ & \multicolumn{3}{|c|}{ Detection times (Hours) } \\
\cline { 2 - 5 } & & Max & Min & $\mathbf{1 9 . 3 3}$ \\
\hline $\begin{array}{c}\text { All Gram Positive } \\
\text { Bacteria }\end{array}$ & $\mathbf{2 0}$ & - & $\mathbf{7 . 3}$ & $\mathbf{3 2 . 5 1}$ \\
\hline $\begin{array}{c}\text { Coagulase negative } \\
\text { staphylococcus }\end{array}$ & $7(35)$ & $\mathbf{3 8}$ & $\mathbf{2 . 2}$ & $\mathbf{1 3 . 2 3}$ \\
\hline Staphylococcus aureus & & & $\mathbf{3 . 2}$ & $\mathbf{1 0 . 0 5}$ \\
\hline Streptococcus pneumonia & $6(30)$ & $\mathbf{4 3}$ & $\mathbf{1 2 . 3}$ & $\mathbf{2 1 . 3 2}$ \\
\hline Enterococcus & $2(10)$ & $\mathbf{1 2 . 2 5}$ & $\mathbf{2 4 . 0 4}$ \\
\hline Candida & $2(10)$ & $\mathbf{2 9 . 5 3}$ & $\mathbf{8 2}$ & $\mathbf{1 9 . 0 6}$ \\
\hline All Gram Negative Bacteria & $\mathbf{1 7}$ & - & $\mathbf{8 . 3}$ & $\mathbf{2 2 . 0 7}$ \\
\hline Pseudomonas & $3(17.64)$ & $\mathbf{4 6}$ & $\mathbf{1 2}$ & $\mathbf{1 3 . 3}$ \\
\hline Klebsiella & $3(17.64)$ & $\mathbf{1 5}$ & $\mathbf{1 2}$ & $\mathbf{1 8 . 4 7}$ \\
\hline Acinetobacter & 1 & & $\mathbf{8 . 5}$ & $\mathbf{1 0 . 7 7}$ \\
\hline Citrobacter & $1(5.88 \%)$ & $\mathbf{9 6}$ & $\mathbf{1 3 2}$ \\
\hline Brucella & $1(5.88)$ & & & \\
\hline
\end{tabular}

The mean detection time taken by BACTEC 9050 for gram-positive bacteria and gram-negative bacteria in this study were 19.33 hours and 19.06 hours respectively whereas time taken for detection of Brucella isolates was minimum of 4 days (96hrs) and maximum of 7 (168)days

Table 8: Total time of detection of Bacterial isolates by Conventional blood culture methods

\begin{tabular}{|c|c|c|}
\hline \multirow{2}{*}{ Method } & \multicolumn{2}{|c|}{ Time taken for positivity } \\
\cline { 2 - 3 } & Gram positive bacteria $(\mathbf{n = 2 0})$ & Gram negative bacteria(n=17) \\
\hline Conventional blood culture & $5-7$ Days & $5-7$ Days \\
\hline
\end{tabular}

Total time taken for detection of bacterial isolates by conventional methods was upto 5-7 days with repeated subcultures.

Table 9: Distribution of Pathogenic isolates by Conventional blood culture and BACTEC 9050.

\begin{tabular}{|l|c|c|}
\hline \multicolumn{1}{|c|}{ Pathogenic isolates $(\mathbf{n})$} & Conventional blood culture (n) & BACTEC 9050 \\
\hline Coagulase negative Staphylococcus (7) & 6 & 7 \\
\hline Staphylococcus aureus (6) & 4 & 6 \\
\hline Streptococcus pneumonia (3) & 2 & 3 \\
\hline Enterococcus (2) & 1 & 2 \\
\hline Candida (2) & 2 & 2 \\
\hline
\end{tabular}


Comparative Evaluation of Conventional Blood Culture with Bactec 9050 for Bacterial Isolates.....

\begin{tabular}{|l|c|c|}
\hline Salmonella typhi (4) & 2 & 4 \\
\hline E.coli (4) & 2 & 4 \\
\hline Pseudomonas (3) & 2 & 3 \\
\hline Klebsiella(3) & 2 & 1 \\
\hline Acinetobacter (1) & 1 & 1 \\
\hline Citrobacter (1) & 1 & 1 \\
\hline Brucella (1) & - & 2 \\
\hline
\end{tabular}

Maximum pathogenic isolates detected by both conventional blood culture and Bactec 9050 were coagulase negative Staphylococcus and Staphylococcus aureus. The highest rate of recovery of pathogenic isolates was by BACTEC 9050 i.e. 100\% (37/37) as compared to conventional blood culture methods $67.56 \%$ (25/37). There were 12 samples (32\%) which were found to be positive only by BACTEC 9050 and not by Conventional blood culture methods. One positive sample of Brucella (3\%) was detected by BACTEC 9050 only.

Table 10: Efficacy of BACTEC 9050 with respect to Conventional blood culture methods

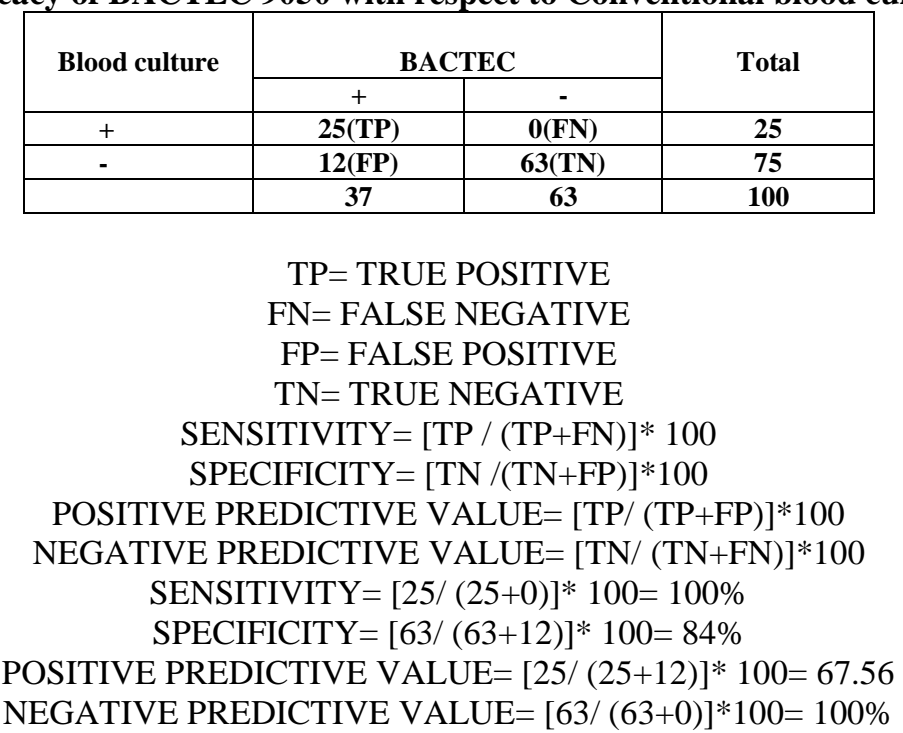

The Sensitivity,Specificity,Positive Predictive value and Negative predictive value of BACTEC 9050 was found to be $100 \%, 84 \%, 67.56 \%$ and $100 \%$ respectively against conventional culture.

\section{Discussion}

Patients with fever of unknown origin (FUO) are elusive and challenging clinical cases. Timely detection and identification of blood borne pathogen is one of the most important functions of microbiology laboratory. In the present study, blood culture positivity was seen in $46 \%$ cases with $37 \%$ pathogenic isolates comprising of $54.05 \%$ gram positive and $45.94 \%$ gram negative bacteria. These results are consistent with the study done by Jung et al (1999) ${ }^{7}$ and Handa et al who reported $43.8 \%$ infectious causes of FUO. ${ }^{14}$ In present study, maximum isolates of gram positive bacteria were coagulase negative Staphylococcus (35\%) followed by Staphylococcus aureus (30\%), Streptococcus pneumonia (15\%), Enterococcus (10\%) and Candida (10\%). While gram negative bacteria comprised mainly of Salmonella typhi $(29.41 \%)$ followed by E coli $(17.64 \%)$, Pseudomonas (17.64\%), Klebsiella (17.64\%), Acinetobacter (5.88\%), Citrobacter (5.88\%) and Brucella (5.88\%). These findings are in agreement with study done by Gopi et al (2011) who reported that among $(61.52 \%)$ gram positive bacteria, maximum were coagulase negative Staphylococcus (29.92\%) while gram negative bacteria (36.94\%) comprised mainly of Enterobacteriacea (25.34\%) i.e. Salmonella typhi were $(14.35 \%)$, E coli $(2.90 \%)$, Klebsiella (1.06\%), Citrobacter $(1.37 \%)$ and Acinetobacter $(5.80 \%) .{ }^{15}$ However contrary to present study, Durmaz et al (2003) reported more gram negative isolates from such FUO cases. ${ }^{8}$ In present study, males constituted majority (65.22\%) of the patients from rural background (65.22\%). No definite correlation between occupation and culture positivity was seen. This finding was similar with Asmaa et al (2005) who reported $(66.66 \%)$ male and $(33.33 \%)$ female and found male to female ratio of $1.99: 1^{16}$. The increase member of male patients over female in this study might be due to occupational exposure to animals. Male are the active and main earning member of the most of the family still now, so they are more privileged to visit physician chamber for treatment. ${ }^{17}$ Maximum patients were found in the younger age group of less than 20 years $(52.17 \%)$ followed by $31-40$ years $(21.73 \%), 21-30$ years $(13.04 \%), 31-40$ years( $8.69 \%)$ and $(4.34 \%)$ 
were $>50$ years of age. A study by Asmaa et al (2005) reported most of the clinically suspected cases (43.30\%) in the age group between 1-15 years having very close correlation with present study ${ }^{16}$. This study evaluates the capability, efficiency and reliability of BACTEC 9050 in comparison to conventional blood culture for detection of bacterial isolates. The highest rate of recovery of pathogenic isolates was by BACTEC 9050 i.e. 37 (100\%) as compared to conventional blood culture methods 25 out of $37(67.56 \%)$. There were 12 samples $(32 \%)$ which were found to be positive only by BACTEC 9050 and not by conventional blood culture methods. Positive sample of Brucella 1(3\%) was detected by BACTEC 9050 only. The mean detection time taken by BACTEC 9050 for gram-positive bacteria and gram-negative bacteria in this study was 19.33 hours and 19.06 hours respectively whereas time taken for detection of Brucella isolates was minimum of 4 days and maximum of 7 days. Conventional methods took upto 5-7 days to detect positive bacterial isolates with repeated subcultures. These results are consistent with the study done by Gopi et al $(2011)^{15}$ who reported that the mean detection time for the clinical significant isolates by BACTEC 9050 was $21 \mathrm{~h}$ with $9 \%$ pathogenic positive cultures comprising of $61 \%$ gram- positive and 36\% gram-negative bacteria. Hence BACTEC 9050 was found to be more sensitive in detecting pathogenic bacterial isolates as compared to conventional blood culture methods.

\section{Conclusion}

In the end it is concluded that out of 100 samples, $46 \%$ were culture positive and $54 \%$ were sterile by either of the two methods i.e. conventional blood culture and BACTEC 9050. Highest rate of pathogenic isolates were recovered by BACTEC $9050(100 \%)$ as compared to conventional blood culture (67.56\%) indicating sensitivity and accuracy of BACTEC 9050 for culturing the microorganism in clinical specimens. Futhermore, mean time to detection of significant pathogens was significantly less with the BACTEC 9050 than with conventional media. $80.43 \%$ pathogenic isolates were detected with $(54.05 \%)$ gram positive and $(45.94 \%)$ gram negative isolates. Maximum gram positive isolates were coagulase negative Staphylococcus (35\%) and Staphylococcus aureus (30\%) while gram negative bacteria comprised mainly of Salmonella typhi $(29.41 \%)$ and E coli $(17.64 \%)$. Hence BACTEC 9050 has high sensitivity, specificity, PPV and NPV as compared to conventional blood culture examination..

\section{References}

[1]. Molavi A, Weinstein L. Persistent perplexing pyrexia: some comments on etiology and diagnosis. Med Clin North Am. 1970;54: 379-396.

[2]. Petersdorf RG, Beeson PB. Fever of unexplained origin: Report on 100 Medicine cases. Medicine.1961; 40:130.

[3]. Arnow PM, Flaherty JP. Fever of unknown origin. Lancet.1997; 350:575-580.

[4]. Mansueto P, Lorenzo G, Rizzo M. Fever of unknown origin in a Mediterranean survey from a division of internal medicine: report of 91 cases during a 12-year-period. Intern Emerg Med. 2008; 56:456-475.

[5]. Ergonul O, Willke A, Azap A. Revised definition of 'fever of unknown origin': limitations and opportunities. J Infect. 2005 Jan; 50(1):1-5.

[6]. De Kleijn EM, Vanderbroucke JP, Vander Meer JW. Fever of unknown origin (FUO). A prospective multicenter study of 167 patients with FUO, using fixed epidemiologic entry criteria. Medicine. 1997; 76:392-400.

[7]. Jung A, Singh MM, Jajoo U. Unexplained fever- analysis of 233 cases in a referral hospital. Indian J Med Sci.1999; 53:535-544.

[8]. Durmaz, G., Us, T., Aydinli, A. Optimum detection times for bacteria and yeast species with the BACTEC 9120 aerobic blood culture system: Evaluation for a 5-year period in a Turkish University Hospital. J. Clin. Microbiol. 2003 ; 41: $819-821$.

[9]. Dokuzoguza. B, Ergonula O, Baykam, N.Characteristics of B. melitensis Versus B. Abortus bacteraemias. J. Infect. 2005; 50:4155.

[10]. BACTEC 9050 System Users Manual. Document Number MA-0103. BD Biosciences.

[11]. BACTECTM PLUS Aerobic/F and PLUS Anaerobic/F Culture Vials Insert Rev. PP-088E BD Biosciences.

[12]. BACTECTM PEDS PLUS/F Culture Vials Insert.Rev. PP-091-I. BD Biosciences.

[13]. J.G Collee, W.Marr. Culture of Bacteria. Practical Medical Microbiology Mackie and McCartney 14 ${ }^{\text {th }}$ edition,Elsevier.2008:123124.

[14]. Handa R, Singh S, Singh N, Wali JP. Brucellosis in north India, a prospective study. J Commun Dis. 1998; 30(2): 85-7.

[15]. Gopi Anjana, Ravikumar KL, Ambarish MG, Shwethalatha NN, Shree Keerthi, Ashwini KV, Arpita S, Debjani C. Time to Positivity of Microorganisms with BACTEC 9050. An 18-month Study Among Children of 28 Days to 60 Months in an South Indian Tertiary Hospital. International Journal of Microbiological Research.2011;2(1):12-17.

[16]. Asmaa AAH, Amal SMS, Mohamed A. 2005,.Seroepidemiology Study of Human Brucellosis in Assiut Goverorate. The Egyptian Journal of Immunology. 2005; 12(1), 49-56.

[17]. Salari MH. Seroepidemiological Survey of Brucellosis among Animal Farmers of Yazd Province. Iranian J. Publ. Health.2002; 31(1-2), 29-32. 